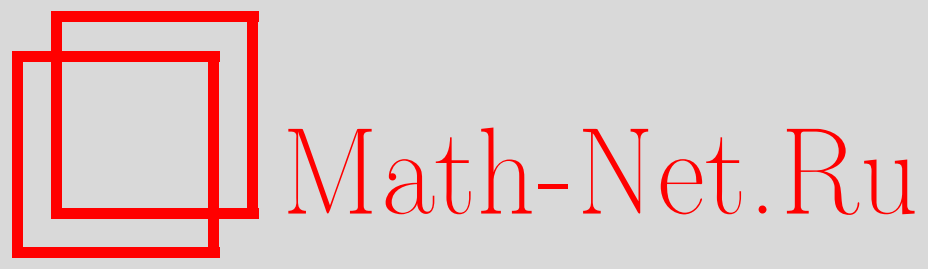

А. А. Досиев, Теорема о представлении локальных операторных пространств, Функи. анализ и его прил., 2007, том 41, выпуск 4, 73-78

DOI: https://doi.org/10.4213/faa2881

Использование Общероссийского математического портала Math$\mathrm{Net.Ru}$ подразумевает, что вы прочитали и согласны с пользовательским соглашением

http://www . mathnet.ru/rus/agreement

Параметры загрузки:

IP : 54.237 .59 .107

26 апреля 2023 г., $17: 23: 51$

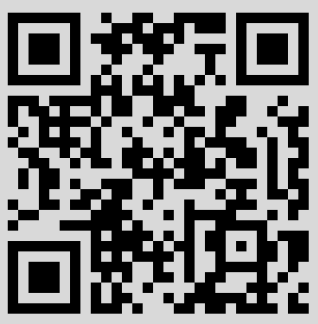


УДК 517.98

\title{
Теорема о представлении локальных операторных пространств
}

\author{
(c) 2007. А. Досиев
}

Известная теорема о представлении операторных пространств [3, теорема 2.3.5] утверждает, что каждое абстрактное операторное пространство $V$ может быть реализовано как подпространство пространства $\mathscr{B}(H)$ всех ограниченных линейных операторов в гильбертовом пространстве $H$. Под реализацией понимается матричная изометрия $\Phi: V \rightarrow \mathscr{B}(H)$ пространства $V$ на подпространство $\Phi(V) \subseteq \mathscr{B}(H)$. Этот результат играет центральную роль в теории операторных пространств и позволяет дать абстрактную характеризацию любого линейного пространства ограниченных линейных операторов в гильбертовом пространстве. С физически мотивированной точки зрения операторные пространства можно представлять себе как квантованные нормированные пространства, в которых функции заменяются на операторы (при этом классические нормированные пространства рассматриваются как абстрактные функциональные пространства). Другая мотивировка подсказывается свойством доминирования, отмеченным в задаче о некоммутативном функциональном исчислении [1, разд. 4], которое подтверждает, что (совместные) спектральные свойства элементов операторной алгебры, возможно, выражаются в терминах матриц над исходной алгеброй. Реализация этого предложения привела бы к разумной совместно-спектральной теории в операторной алгебре. Чтобы подвести более солидное обоснование под квантовую физику и некоммутативную теорию функций, необходимо рассматривать операторные аналоги локально выпуклых пространств, т. е. квантования таковых. А именно, можно рассматривать линейные пространства неограниченных операторов в гильбертовых пространствах или, более общим образом, проективные пределы операторных пространств. В последние годы эта теория развивалась Әффросом и Уэбстером [4] под названием «локальные операторные пространства». Центральным и весьма тонким результатом их исследований является операторная версия классической биполярной теоремы [4, предложение 4.1].

Цель настоящей заметки состоит в том, чтобы дать внутреннее описание локальных операторных пространств, подобное упомянутой выше характеризации операторных пространств. Мы показываем, что любое локальное операторное пространство может быть реализовано как подпространство неограниченных операторов в гильбертовом пространстве. При этом если в заданном локальном операторном пространстве имеется ограниченная локально выпуклая топология, то оно может быть реализовано ограниченными операторами в гильбертовом пространстве. Этот результат обобщает теорему Руана о представлении операторных пространств. Чтобы восстановить естественные связи между локальными операторными пространствами и унитальными мультинормированными $C^{*}$-алгебрами, как это было в нормированном случае, мы вводим локальные операторные системы, мотивированные теоремой о представлении локальных операторных пространств. Известный класс унитальных мультинормированных $C^{*}$-алгебр, называемых $O p^{*}$-алгебрами [7], [6, п. 3.2.3], представляет 
собой яркий пример локальных операторных систем. Центральную роль для таких систем играет понятие локальной положительности. В терминах локальной положительности мы доказываем для этих систем теорему Арвесона-ХанаБанаха-Уэбстера, в которой вместо $\mathscr{B}(H)$ фигурирует максимальная операторная $C^{*}$-алгебра Фреше.

Далее мы предлагаем локально выпуклую версию теоремы Стайнспринга для отображения унитальной мультинормированной $C^{*}$-алгебры в локальную операторную систему, которая, в свою очередь, приводит к теореме о представлении для унитальных мультинормированных $C^{*}$-алгебр. Наконец, мы переносим известный результат об инъективности из [2] на случай локальных операторных систем.

Я хочу поблагодарить М. Фрагулопулу, А. Геондеа, Ю. Туровского, А. Пирковского и К. Уэбстера за полезные обсуждения подробностей настоящей работы. Я также благодарен рецензенту за ценные замечания.

1. Пусть $X$ - линейное пространство, и пусть заданы полунормы $p^{(n)}: M_{n}(X)$ $\rightarrow[0, \infty)$ на пространствах $M_{n}(X) n \times n$-матриц над $X$. Семейство $p=\left\{p^{(n)}: n \in\right.$ $\mathbb{N}\}$ называется матричной полунормой [4] на $X$, если оно обладает свойствами

M1. $p^{(m+n)}(v \oplus w) \leqslant \max \left\{p^{(m)}(v), p^{(n)}(w)\right\}$,

M2. $p^{(n)}(\alpha v \beta) \leqslant\|\alpha\| p^{(m)}(v)\|\beta\|$

для всех $v \in M_{m}(X), w \in M_{n}(X), \alpha \in M_{n, m}, \beta \in M_{m, n}$, где $v \oplus w-$ прямая сумма элементов $v$ и $w$, а $M_{n, m}=M_{n, m}(\mathbb{C})$ - пространство всех $n \times m$ матриц со скалярными элементами. Линейное пространство $X$ называется ( $a б$ страктным) операторным пространством [3, разд. 2.1], если оно снабжено матричной нормой. Линейное пространство $X$, снабженное семейством матричных полунорм $\left\{p_{\alpha}: \alpha \in \Lambda\right\}$, называется абстрактным локалъным операторным пространством. Говорят, что полинормированное пространство $X$ имеет ограниченную топологию, если в нем есть ограниченнная бочка. В частности, бочечное полинормированное пространство нормируемо в своей ограниченной топологии.

Если $X$ - локальное операторное пространство с ограниченной полинормированной топологией, то говорят, что $X$ - локально ограниченное операторное пространство. Линейному отображению $\varphi: X \rightarrow Y$ линейных пространств $X$ и $Y$ соответствует линейное отображение $\varphi^{(n)}: M_{n}(X) \rightarrow M_{n}(Y)$, $\varphi^{(n)}\left(\left[x_{i j}\right]\right)=\left[\varphi\left(x_{i j}\right)\right]$, для каждого $n \in \mathbb{N}$. Пусть $X$ и $Y$ - локальные операторные пространства с (насыщенными) семействами матричных полунорм $\left\{p_{\alpha}\right\}_{\alpha \in \Lambda}$ и $\left\{q_{\iota}\right\}_{\iota \in \Omega}$ соответственно. Говорят, что линейное отображение $\varphi: X \rightarrow Y$ мamрично непрерьвно, если для любого $\iota \in \Omega$ существуют такие $\alpha \in \Lambda$ и положительная постоянная $C_{\iota \alpha}$, что $q_{\iota}^{(n)} \varphi^{(n)} \leqslant C_{\iota \alpha} p_{\alpha}^{(n)}$ для всех $n$. Если $C_{\iota \alpha} \leqslant 1$ для всех возможных $\iota$ и $\alpha$, то говорят, что $\varphi-$ матричное сжатие. Далее, инъективное отображение $\varphi$ называется матричной изометрией, если $\Omega=\Lambda$ и $q_{\alpha}^{(n)} \varphi^{(n)}=p_{\alpha}^{(n)}$ для всех $\alpha$ и $n$.

2. Введем локальные направленности для подпространства, состоящего из неограниченных операторов. Зафиксируем гильбертово пространство Н. Пусть $\mathfrak{L}(H)$ - множество всех неограниченных операторов в $H$. Рассмотрим подмножество $V \subseteq \mathfrak{L}(H)$, все элементы которого имеют одну и ту же область определения $E$, представляющую собой плотное подпространство в $H$, причем $T(E) \subseteq E$ 
для всех $T \in V$. Будем говорить, что $V$ - подпространство (ассоииативная подалгебра) неограниченных операторов в $H$, если $V$ замкнуто относительно обычных алгебраических операций, определенных для элементов из $V$. Пространство $E$ называется носителем подпространства (подалгебры) $V$ и обозначается через $\operatorname{supp}(V)$. Если $\operatorname{supp}(V)$ замкнуто, то $V$ есть просто линейное подпространство (или ассоциативная подалгебра) алгебры $L(H)$ всех линейных преобразований пространства $H$.

Пусть теперь $\mathfrak{p}=\left\{P_{\alpha}\right\}$ - направленность, состоящая из ортогональных проекторов в $\mathfrak{L}(H)$, и пусть $H_{\alpha}=\operatorname{im}\left(P_{\alpha}\right)$. Множество $\left\{H_{\alpha}\right\}$ представляет собой фильтрованное вверх семейство замкнутых подпространств в $H$. Поэтому их объединение $\bigcup_{\alpha} H_{\alpha}$ является линейным подпространством в $H$.

Определение А. Пусть $V$ - подпространство неограниченных операторов в гильбертовом пространстве $H$, и пусть $p=\left\{P_{\alpha}\right\}$ - направленность из ортогональных проекторов в $\mathfrak{L}(H)$, такая, что $\operatorname{supp}(V)=\bigcup_{\alpha} H_{\alpha}$, где $H_{\alpha}=\operatorname{im}\left(P_{\alpha}\right)$.

Будем говорить, что $\mathfrak{p}$ - локальная направленность для $V$, если $P_{\alpha} T=T P_{\alpha}$ $($ на $\operatorname{supp}(V))$ и $P_{\alpha} T P_{\alpha} \in \mathscr{B}(H)$ для всех $T \in V$ и $P_{\alpha} \in \mathfrak{p}$. Далее, $\mathfrak{p}$ называется слабой локальной направленностью для $V$, если все подпространства $H_{\alpha}$ инвариантны относительно $V$ и $V \mid H_{\alpha} \subseteq \mathscr{B}\left(H_{\alpha}\right)$ для всех $\alpha$.

Заметим, что в этом случае носитель $\operatorname{supp}(V)$ можно рассматривать как строгий индуктивный предел направленного семейства гильбертовых пространств $\mathscr{E}=\left\{H_{\alpha}: \alpha \in \Lambda\right\}$, т. е. $\operatorname{supp}(V)=\lim _{\mathfrak{E}}[5$, разд. 4.3]. При этом каждый оператор $T \in V$ определяет на носителе линейный оператор $T_{0}=T \mid \operatorname{supp}(V)$, такой, что каждое подпространство $H_{\alpha}$ инвариантно относительно $T_{0}$ и $T_{0} \mid H_{\alpha} \in \mathscr{B}\left(H_{\alpha}\right)$. Поэтому оператор $T_{0}$ непрерывен на $\operatorname{supp}(V)$.

Зафиксируем теперь число $n \in \mathbb{N}$ и рассмотрим $n$-ю гильбертову степень $H^{n}$ пространства $H$. Если $V$ - подпространство неограниченных операторов в $H$, то пространство матриц $M_{n}(V)$ можно канонически отождествить с некоторым подпространством неограниченных операторов в $H^{n}, M_{n}(V) \subseteq \mathfrak{L}\left(H^{n}\right), n \in \mathbb{N}$. При этом $\operatorname{supp}\left(M_{n}(V)\right)=\operatorname{supp}(V)^{n}$. Если $T \in \mathfrak{L}(H)$, то через $T^{\oplus n} \in \mathfrak{L}\left(H^{n}\right)$ обозначим оператор $\left(x_{i}\right)_{i} \mapsto\left(T x_{i}\right)_{i}$. Положим $\mathfrak{p}^{\oplus n}=\left\{P_{\alpha}^{\oplus n}\right\}$ для направленности ортогональных проекторов $\mathfrak{p}=\left\{P_{\alpha}\right\} \subseteq \mathfrak{L}(H)$. Тогда $\mathfrak{p}^{\oplus n}-($ слабая) локальная направленность для подпространства $M_{n}(V) \subseteq \mathfrak{L}\left(H^{n}\right)$, если $\mathfrak{p}$ является таковой для $V$. Конкретное операторное пространство $V$ определяется как подпространство пространства $\mathscr{B}(H)$ для некоторого гильбертова пространства $H$ (см. [3, разд. 2.1]). Включения $M_{n}(V) \subseteq M_{n}(\mathscr{B}(H))=\mathscr{B}\left(H^{n}\right)$, $n \in \mathbb{N}$, определяют матричную норму на $V$. В общем случае пусть $V \subseteq \mathfrak{L}(H)$ - подпространство неограниченных операторов, а $\mathfrak{p}=\left\{P_{\alpha}\right\}_{\alpha \in \Lambda}$ - (слабая) локальная направленность для $V$. Зафиксируем какое-то $\alpha \in \Lambda$. Тогда пространство $H_{\alpha}^{n}\left(=\operatorname{im}\left(P_{\alpha}^{\oplus n}\right)\right)$ инвариантно относительно всех операторов $T \in M_{n}(V)$. Положим $p_{\alpha}^{(n)}(T)=\left\|T \mid H_{\alpha}^{n}\right\|, \alpha \in \Lambda$. Таким образом, $\mathfrak{p}^{(n)}=\left\{p_{\alpha}^{(n)}\right\}_{\alpha \in \Lambda}-$ возрастающее семейство полунорм на $M_{n}(V)$. Заметим, что если $\operatorname{supp}(V)=H$ и $\mathfrak{p}$ - локальная направленность, то $V \subseteq \mathscr{B}(H)$ и $\mathfrak{p}^{(n)}$ ограничено. При этом $\sup \mathfrak{p}^{(n)}$ сводится к обычной операторной норме в $M_{n}(V) \subseteq \mathscr{B}\left(H^{n}\right)$. Положим $p_{\alpha}=\left\{p_{\alpha}^{(n)}\right\}_{n \in \mathbb{N}}$ и $\mathfrak{P}=\left\{p_{\alpha}\right\}_{\alpha \in \Lambda}$. Тогда $p_{\alpha}$ - матричная полунорма на $V$. Таким образом, если задана (слабая) локальная направленность для подпространства $V \subseteq \mathfrak{L}(H)$, то мы автоматически снабжаем $V$ матричной топологией, порожденной семейством матричных полунорм $\mathfrak{P}$. В этом случае будем говорить, что 
$V$ - конкретное локальное операторное пространство. Если $\operatorname{supp}(V)=H$, а $\mathfrak{p}$ - локальная направленность для $V$, то на $V$ корректно определена матричная норма $p=\sup _{\alpha} p_{\alpha}=\sup \mathfrak{P}$, так что $V$ оказывается локально ограниченным операторным пространством. Это пространство называется конкретным локально ограниченным операторным пространством. Под реализачией локального (или локально ограниченного) операторного пространства $V$ на гильбертовом пространстве $H$ будем понимать такое отображение $\varphi: V \rightarrow \mathfrak{L}(H)$, что $\varphi(V)-$ конкретное локальное операторное пространство на $H$, а $\varphi$ - матричная изометрия пространства $V$ на $\varphi(V)$.

Теорема 1. Пусть $V-($ хаусдорфово) локальное операторное пространство. Тогда существует реализачия $\varphi: V \rightarrow \mathfrak{L}(H)$ пространства $V$ на гильбертовом пространстве $H$. При этом если $V$ - локально ограниченное операторное пространство, то существует такая его реализащия $\varphi: V \rightarrow \mathscr{B}(H)$, что $\varphi(V)$ - конкретное локально ограниченное операторное пространство.

Заметим, что если, например, $V$ - локальное операторное пространство Фреше, то слабая локальная направленность для подпространства $\varphi(V)$ оказывается локальной направленностью.

3. Пусть $V \subseteq \mathfrak{L}(H)$ - конкретное локальное операторное пространство с локальной направленностью $\mathfrak{p}=\left\{P_{\alpha}\right\}_{\alpha \in \Lambda}$. Тогда каждому оператору $T \in V$ соответствует двойственный неограниченный оператор $T^{\prime} \in \mathfrak{L}(H)$, такой, что $\operatorname{supp}(V) \subset \operatorname{dom}\left(T^{\prime}\right)$ и $T^{\prime} \mid H_{\alpha}=\left(T \mid H_{\alpha}\right)^{*}$ для всех $\alpha$. Пусть $T^{*}=\left.T^{\prime}\right|_{\operatorname{supp}(V)}$. Таким образом, $V^{*}=\left\{T^{*}: T \in V\right\}-$ подпространство неограниченных операторов в $H$ с одним и тем же носителем $\operatorname{supp}(V)$, a $\mathfrak{p}-$ локальная направленность также и для $V^{*}$.

Определение В. (Конкретное) локальное операторное пространство $V \subseteq$ $\mathfrak{L}(H)$ с локальной направленностью $\mathfrak{p}$ называется локальной операторной системой на $H$, если $V=V^{*}$ и $I_{H} \in V$, где $I_{H}$ - тождественный оператор в $\operatorname{supp}(V)$. Подпространство $W \subseteq V$ называется операторной подсистемой, если $W=W^{*}$ и $I_{H} \in W$. Далее, под локальной операторной $C^{*}$-алгеброй $A$ на пространстве $H$ с направленностью $\mathscr{E}=\left\{H_{\alpha}\right\}_{\alpha \in \Lambda}$ мы понимаем ассоциативную подалгебру $A \subseteq \mathfrak{L}(H)$, которая является полной локальной операторной системой на $H$ с локальной направленностью $\mathfrak{p}$.

Заметим, что топологическое пополнение $\widetilde{V}$ локальной операторной системы - снова локальная операторная система на $H$ с той же локальной направленностью, что и у $V$. Если, кроме того, $V$ - ассоциативная подалгебра в $\mathfrak{L}(H)$, то этим же свойством обладает и $\widetilde{V}$. Далее, каждая локальная операторная $C^{*}$-алгебра есть унитальная мультинормированная $C^{*}$-алгебра относительно топологии локальной операторной системы. Операторная $C^{*}$-алгебра Фреше определяется как операторная $C^{*}$-алгебра на гильбертовом пространстве $H$ со счетной направленностью $\mathscr{E}=\left\{H_{n}\right\}_{n \in \mathbb{N}}$. Под максимальной операторной $C^{*}$-алгеброй на $H$ с направленностъю $\mathscr{E}$ мы понимаем множество $\mathfrak{B}_{\mathscr{E}}$ всех неограниченных операторов $T \in \mathfrak{L}(H)$, таких, что $\bigcup \mathscr{E}=\operatorname{dom}(T), P_{\alpha} T \subset T P_{\alpha}$ и $P_{\alpha} T P_{\alpha} \in \mathscr{B}(H)$ для всех $\alpha \in \Lambda$. Например, такова алгебра $\mathscr{B}(H)$ с направленностью $\mathscr{E}=\{H\}$.

Пусть $V$ - локальная операторная система на $H$ с локальной направленностью $\mathfrak{p}=\left\{P_{\alpha}\right\}_{\alpha \in \Lambda}$. Говорят, что элемент $T \in V$ локально положителен, если 
$T \mid H_{\alpha} \geq 0\left(\right.$ в $\left.\mathscr{B}\left(H_{\alpha}\right)\right)$ для некоторого $\alpha \in \Lambda$. Аналогично, если $A-$ унитальная мультинормированная $C^{*}$-алгебра с фиксированным определяющим семейством $C^{*}$-полунорм $\left\{p_{\alpha}\right\}_{\alpha \in \Lambda}$, то элемент $a \in A$ локально положителен, если $a=b^{*} b+x$ для некоторого $x \in A$, такого, что $p_{\alpha}(x)=0$. Пусть $V \subseteq \mathfrak{L}(H)$ и $W \subseteq \mathfrak{L}(K)$ - локальные операторные системы с локальными направленностями $\mathfrak{p}=\left\{P_{\alpha}\right\}_{\alpha \in \Lambda}$ и $\mathfrak{q}=\left\{Q_{\iota}\right\}_{\iota \in \Omega}$ соответственно. Линейное отображение $\varphi: V \rightarrow W$ называется локально вполне положительным, если любому элементу $\iota \in \Omega$ соответствует такой элемент $\alpha \in \Lambda$, что $\varphi^{(n)}(v) \mid K_{\iota}^{n} \geq 0$ для любого $v \in M_{n}(V)$, удовлетворяющего условию $v \mid H_{\alpha}^{n} \geq 0$, и $\varphi^{(n)}(v) \mid K_{\iota}^{n}=0$, если $v \mid H_{\alpha}^{n}=0$, для любого $n$, где $H_{\alpha}=\operatorname{im}\left(P_{\alpha}\right)$ и $K_{\iota}=\operatorname{im}\left(Q_{\iota}\right)$. Будем называть отображение $\varphi: V \rightarrow W$ морфизмом, если оно унитально и локально вполне положительно. Локальное операторное пространство (соответственно система) $V$ называется инъективной, если для каждого операторного подпространства (соответственно операторной подсистемы) $W_{0} \subseteq W$ локального операторного пространства (соответственно системы) $W$ каждое матричное сжатие (соответственно каждый морфизм) $\psi: W_{0} \rightarrow V$ может быть продолжен до матричного сжатия (соответственно морфизма) $\bar{\psi}: W \rightarrow V$. По теореме Арвесона-Хана-Банаха [8, п. 2.31] $\mathscr{B}(H)$ является инъективным локальным операторным пространством.

Сформулируем теперь теорему Арвесона-Хана-Банаха-Уэбстера для локальных операторных систем.

Теорема 2. Пусть $V$ - локальная операторная система в $H, W$ - ее операторная подсистема, а $\mathfrak{B}_{\mathscr{E}}$ - максимальная операторная $C^{*}$-алгебра Фреше. Тогда для любого локально вполне положительного отображсения $\varphi: W \rightarrow$ $\mathfrak{B}_{\mathscr{E}}$ существует локально вполне положительное продолжсене $\Phi: V \rightarrow \mathfrak{B}_{\mathscr{E}}$. $B$ частности, $\mathfrak{B}_{\mathscr{E}}-$ инъективная локальная операторная система.

Используя теоремы 1 и 2, можно установить, что для операторной системы Фреше ее инъективность в смысле локальных операторных пространств эквивалентна инъективности в смысле локальных операторных систем.

Локально выпуклая версия теоремы Стайнспринга имеет следующий вид.

Теорема 3. Пусть $A$ - унитальная мультинормированная $C^{*}$-алгебра, $V$ - локальная операторная система на гильбертовом пространстве $H, a$ $\varphi: A \rightarrow V$ - матричное сжнатие. Если $\varphi$ локально вполне положительно, то существуют локальная операторная $C^{*}$-алгебра $B$ на гильбертовом пространстве $K$, сжатие $T: H \rightarrow K$ и унитальный сжимающий *-гомоморфизм $\pi: A \rightarrow B$, maкuе, что $T(\operatorname{supp}(V)) \subseteq \operatorname{supp}(B) u$

$$
\varphi(a)=T^{*} \pi(a) T \quad \text { на } \operatorname{supp}(V)
$$

для всех $а \in A$. Если при этом ч унитально, то $T$ - изометрия.

Следствие 4. Пусть $A-$ мультинормированная $C^{*}$-алгебра. Тогда, с точностью до изометрического *-изоморфизма, А является локальной операторной $C^{*}$-алгеброй на гильбертовом пространстве.

Следствие 5. Пусть $A$ u $B$ - унитальные мультинормированные $C^{*}$-алгебры, и пусть $\varphi: A \rightarrow B-$ - унтальный линейный изоморфизм. Если $u$ $\varphi, u \varphi^{-1}$ локально вполне положительны, то $\varphi-$ топологический *-изомор-

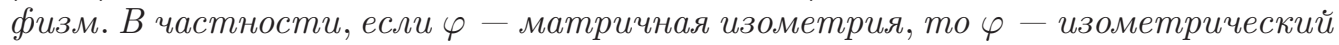
*-изоморфизм.

Локально выпуклая версия внедиагонального приема Паульсена, основанная на теоремах 2 и 3, позволяет доказать следующий результат. 
Теорема 6. Пусть $A-$ унитальная мультинормированная $C^{*}$-алгебра, $\mathfrak{B}_{\mathscr{E}}$ - максимальная операторная $C^{*}$-алгебра Фреше на гилъбертовом пространстве $H$, а $\varphi: A \rightarrow \mathfrak{B}_{\mathscr{E}}$ - матричное сжатие. Тогда существуют унитальный сжимающий *-гомоморфизм $\pi: A \rightarrow D$ алгебры $A$ в операторную $C^{*}$-алгебру $D$ на гилъбертовом пространстве $X$ и сжатия $H \stackrel{T}{\longrightarrow} X \stackrel{S}{\longrightarrow} H$, такие, что $T(\operatorname{supp}(B)) \subseteq \operatorname{supp}(D) u$

$$
\varphi(a)=S \pi(a) T \quad н a \operatorname{supp}\left(\mathfrak{B}_{\mathscr{E}}\right),
$$

для всех $a \in A$.

Пусть теперь $V$ - инъективная локальная операторная система на пространстве $H$ с локальной направленностью $\mathfrak{p}=\left\{P_{\alpha}\right\}_{\alpha \in \Lambda}$, и пусть $H_{\alpha}=\operatorname{im}\left(P_{\alpha}\right)$, $\alpha \in \Lambda$. Тогда $V$ - операторная подсистема максимальной локальной операторной $C^{*}$-алгебры $\mathfrak{B}_{\mathscr{E}}$ на том же пространстве $H$ с направленностью $\mathscr{E}=$ $\left\{H_{\alpha}\right\}_{\alpha \in \Lambda}$. Матричная топология на $V$ (соответственно на $\mathfrak{B}_{\mathscr{E}}$ ) определяется семейством полунорм $\left\{p_{\alpha}\right\}_{\alpha \in \Lambda}$, где $p_{\alpha}^{(1)}(b)=\left\|b \mid H_{\alpha}\right\|_{\mathfrak{B}_{\mathscr{E}}\left(H_{\alpha}\right)}, b \in \mathfrak{B}_{\mathscr{E}}$. Заметим, что $\operatorname{supp}(V)=\operatorname{supp}\left(\mathfrak{B}_{\mathscr{E}}\right)=\bigcup_{\alpha \in \Lambda} H_{\alpha}$. Тождественное отображение $V \rightarrow V$ расширяется до морфизма-проекции $\Phi: \mathfrak{B}_{\mathscr{E}} \rightarrow \mathfrak{B}_{\mathscr{E}}$ на $V$. Для $T, S \in V$ положим $T \cdot S=\Phi(T S)$. Снабженное этим умножением пространство $V$ оказывается $\widehat{\otimes}^{*}$-алгеброй (т. е. локально выпуклой $*$-алгеброй с совместно непрерывным умножением). Точнее, для каждого $\alpha \in \Lambda$ найдется такое $\beta \in \Lambda$, что $\alpha \leqslant \beta, p_{\alpha}^{(n)}(a) \leqslant \sqrt{p_{\alpha}^{(n)}\left(a^{*} \cdot a\right)} \leqslant p_{\beta}^{(n)}(a)$ и $p_{\alpha}^{(n)}(a \cdot b) \leqslant p_{\beta}^{(n)}(a) p_{\beta}^{(n)}(b)$ для любых $a, b \in M_{n}(V)$. Из этих неравенств легко следует, что $\widehat{\otimes}^{*}$-алгебра $V$ является $C^{*}$-алгеброй, если $V$ - операторная система (в этом случае $p_{\alpha}=p_{\beta}$ для любых $\alpha, \beta)$ [2]. В случае локальной операторной системы на основе теоремы 3 и следствия 5 получаем следующее утверждение.

Теорема 7. Пусть $V$ - ингективная локальная операторная система. Тогда $V$ со своей структурой $\widehat{\otimes}^{*}$-алгебры оказывается унитальной мультинормированной $C^{*}$-алгеброй. Это единственное умножение на $V$, относительно которого пространство $V$ вместе $c$ заданной операцией $*$ и матричной топологией является локальной операторной $C^{*}$-алгеброй. Структура локальной операторной системь на $V$, индуцированная мультинормированной $C^{*}$-алгеброй $V$, совпадает с исходной структурой.

\section{ЛИТЕРАТУРА}

[1] A. A. Dosiev, J. Funct. Anal., 230 (2006), 446-493. [2] M. D. Choi, E. G. Efros, J. Funct. Anal., 24:2 (1977), 156-209. [3] E. G. Effros, Z.-J. Ruan, Operator Spaces, London Math. Soc., Clarendon Press, Oxford, 2000. [4] E. G. Effros, C. Webster, in: Operator Algebras and Applications (Samos 1996), NATO Adv. Sci. Inst. Ser. C Math. Phys. Sci., vol. 495, Kluwer, Dordrecht, 1997, 163-207. [5] A. Grothendieck, Topological Vector Spaces, Notes on Math. and Its Appl., Gordon and Breach, London, 1972. [6] А. Я. Хелемский, Банаховы и полинормированные алгебры: общал теория, представления, гомологии, Наука, M., 1989. [7] G. Lassner, Math. Nachr., 52 (1972), 161-166. [8] C. Webster, Local Operator Spaces and Applications, Ph. D., University of California, Los Angeles, 1997.

Atilim University, Ankara, Turkey e-mail: adosiev@atilim.edu.tr,dosiev@yahoo.com
Поступило в редакцию 16 февраля 2006 г. 